HUTP-97/A002

\title{
More on Chiral-Nonchiral Dual Pairs
}

\author{
Peter Cho* \\ Lyman Laboratory \\ Harvard University \\ Cambridge, MA 02138
}

\begin{abstract}
Expanding upon earlier work of Pouliot and Strassler, we construct chiral magnetic duals to nonchiral supersymmetric electric theories based upon $S O(7), S O(8)$ and $S O(9)$ gauge groups with various numbers of vector and spinor matter superfields. Anomalies are matched and gauge invariant operators are mapped within each dual pair. Renormalization group flows along flat directions are also examined. We find that confining phase quantum constraints in the electric theories are recovered from semiclassical equations of motion in their magnetic counterparts when the dual gauge groups are completely Higgsed.
\end{abstract}

$2 / 97$

* Research supported in part by the National Science Foundation under Grant \#PHY-9218167. 


\section{Introduction}

Although two years have passed since Seiberg's discovery of a dual to SUSY QCD [1], constructing weakly coupled descriptions of strongly interacting $\mathcal{N}=1$ supersymmetric gauge theories remains at present more an art than a science. Magnetic counterparts to other electric theories containing matter fields in only fundamental gauge group representations are qualitatively similar to those for SUSY QCD [2,3]. Such models are sufficiently simple that duals to them can be deduced in the absence of any simplifying tree level superpotentials. However, finding duals to theories with even slightly more complicated matter contents remains an outstanding challenge. Several examples have been worked out in cases where operator chiral rings are truncated by classical equations of motion [1 [10]. A handful more have been identified where the magnetic and electric gauge groups are the same [11 13]. But to date, no basic algorithm has been developed which allows one to systematically establish long distance equivalence classes of different microscopic SUSY gauge theories. Recent work of Vafa and collaborators offers the exciting prospect that $\mathcal{N}=1$ duality fundamentally stems from T-duality within string theory [14,15. Hopefully, these string insights will lead to a deeper understanding of field theory duality. But for now, the subject resembles black magic.

Among the duals which have been uncovered since Seiberg's seminal paper, one particular electric-magnetic pair found by Pouliot is especially interesting [16]. The electric theory is based upon an $S O(7)$ gauge group with $N_{f}$ spinor matter fields, while its magnetic counterpart has an $S U\left(N_{f}-4\right)$ gauge group. 1 As the spinor of $S O(7)$ is real, the electric theory is nonchiral. Yet the matter content in Pouliot's dual belongs to a manifestly chiral representation. Similar chiral-nonchiral dichotomies persist in generalizations of the $S O(7)$ model to $S O(8)$ and $S O(10)$ theories with one spinor and $N_{f}$ vectors [17 [19]. These models possess multiple flat directions which connect them to many other dual pairs. They consequently provide valuable laboratories for probing various aspects of $\mathcal{N}=1$ duality.

In this article, we investigate further examples of chiral-nonchiral dual pairs. More specifically, we construct and analyze duals to $S O(7), S O(8)$ and $S O(9)$ models with $N_{f}$ vectors and either one or two spinors. We must note that all the results which we present here can be derived starting from the Pouliot-Strassler $S O(10)$ theory [18]. So none of the

1 As we do not consider global gauge theory properties in this article, we do not distinguish between $S O\left(N_{c}\right)$ and its covering group $\operatorname{Spin}\left(N_{c}\right)$. 
dualities that we will discuss are fundamentally new. Nevertheless, the particular models that we focus upon and which have not previously been considered in the literature exhibit several interesting features. They also provide useful reference points in the search for duals to even more complicated theories. So they are worth studying in their own right.

Our paper is organized as follows. We describe in some detail our simplest dual to an $S O(7)$ model with one spinor and $N_{f}$ vectors in section 2 . We then discuss qualitatively different aspects of duals to $S O(7), S O(8)$ and $S O(9)$ theories with $N_{f}$ vectors and respectively two 8-dimensional spinors, one 8-dimensional spinor and its conjugate, and one 16-dimensional spinor in sections 3,4 and 5. We pay particular attention to renormalization group flows among these models and to recovering electric theory quantum constraints from semiclassical magnetic equations of motion. Finally, we tie together our results and close with some thoughts on finding new duals in section 6 .

\section{2. $S O(7)$ with $N_{f}$ vectors and one spinor}

We begin our study by considering a supersymmetric gauge theory with symmetry group

$$
G=S O(7)_{\text {local }} \times\left[S U\left(N_{f}\right) \times U(1)_{Y} \times U(1)_{R}\right]_{\text {global }}
$$

and matter content

$$
\begin{aligned}
V_{\mu}^{i} & \sim\left(7 ; \square ;-1,1-\frac{4}{N_{f}}\right) \\
Q^{A} & \sim\left(8 ; 1 ; N_{f}, 0\right) .
\end{aligned}
$$

The hypercharge and R-charge assignments for the vector and spinor superfields are chosen so that both abelian factors in $G$ are anomaly free. Since the 7 and 8-dimensional irreps of $S O(7)$ are real, this theory is nonchiral. It is also asymptotically free so long as its one-loop Wilsonian beta function coefficient 2

$$
b_{0}=\frac{1}{2}\left[3 K(\mathrm{Adj})-\sum_{\substack{\text { matter } \\ \text { reps } \rho}} K(\rho)\right]=14-N_{f}
$$

is positive. The full beta function which governs the running of the physical gauge coupling is then negative [20]. The model's infrared dynamics are consequently nontrivial provided it contains $N_{f}<14$ vector flavors.

2 We adopt the $S O\left(N_{c}\right)$ index values $K($ vector $)=2, K$ (adjoint) $=2 \mathrm{~N}_{\mathrm{c}}-4, K($ spinor $)=2^{\frac{\mathrm{N}_{\mathrm{c}}-6}{2}}$ for $N_{c}$ even and $K$ (spinor) $=2^{\frac{N_{c}-5}{2}}$ for $N_{c}$ odd. 
Generic matter field expectation values break the $S O(7)$ gauge group according to the pattern 21]

$$
S O(7) \stackrel{8}{\longrightarrow} G_{2} \stackrel{7}{\longrightarrow} S U(3) \stackrel{7}{\longrightarrow} S U(2) \stackrel{7}{\longrightarrow} 1 .
$$

This expression illustrates the hierarchy of gauge symmetries realized at progressively longer distance scales assuming that the spinor vev's magnitude is larger than the first vector's which in turn is larger than the second vector's and so on. The symmetry breaking information displayed in (2.4) allows one to readily count the gauge invariant operators that act as coordinates on the moduli space of degenerate vacua for small numbers of vector flavors [16,22]. In Table 1, we list the initial parton matter degrees of freedom, the unbroken color subgroup and the number of matter fields eaten by the superHiggs mechanism as a function of $N_{f}$. The remaining uneaten parton fields correspond to the independent hadrons in the low energy effective theory which label D-flat directions of the $S O(7)$ scalar potential.

\begin{tabular}{|c|c|c|c|c|}
\hline$N_{f}$ & Parton DOF & Unbroken Subgroup & Eaten DOF & Hadrons \\
\hline 0 & 8 & $G_{2}$ & $21-14=7$ & 1 \\
1 & 15 & $S U(3)$ & $21-8=13$ & 2 \\
2 & 22 & $S U(2)$ & $21-3=18$ & 4 \\
3 & 29 & 1 & 21 & 8 \\
4 & 36 & 1 & 21 & 15 \\
\hline
\end{tabular}

Table 1: Number of independent hadron operators

The gauge invariant operators' flavor structures are tightly constrained by counting and symmetry considerations. We first recall that the product of two $S O(7)$ spinors decomposes into a sum over rank- $n$ antisymmetric tensor irreps $[n]$ as

$$
8 \times 8=[0]_{S}+[1]_{A}+[2]_{A}+[3]_{S}
$$

where the "S" and "A" subscripts indicate symmetry and antisymmetry under spinor exchange. Since our model contains just one spinor flavor, we can only form hadrons 
involving spinor products in the symmetric [0] and [3] $(\bmod 4)$ irreps. Vector superfields can be contracted into these bispinor combinations using $S O(7)$ Gamma matrices $\Gamma_{\mu}$ and charge conjugation matrix $C$ as Clebsch-Gordan coefficients. We thus form the composite operators

$$
\begin{aligned}
L & =Q^{T} C Q \sim\left(1 ; 1 ; 2 N_{f}, 0\right) \\
M^{(i j)} & =\left(V^{T}\right)^{i \mu} V_{\mu}^{j} \sim\left(1 ; \text { 口 } ;-2,2-\frac{8}{N_{f}}\right) \\
P^{[i j k]} & =\frac{1}{3 !} Q^{T} Y^{[i} Y^{j} Y^{k]} C Q \sim\left(1 ; \text { 日; } 2 N_{f}-3,3-\frac{12}{N_{f}}\right) \\
R^{[i j k l]} & =\frac{1}{4 !} Q^{T} Y^{[i} Y^{j} Y^{k} Y^{l]} C Q \sim\left(1 ; \text { 日 } ; 2 N_{f}-4,4-\frac{16}{N_{f}}\right) .
\end{aligned}
$$

In order to determine whether these hadrons account for all massless fields in the $S O(7)$ model with four or fewer flavors, we tally their number as a function of $N_{f}$ in Table 2. For $N_{f} \leq 3$, the sum over the $L, M$ and $P$ degrees of freedom agrees with the required number of hadrons listed in Table 1 . On the other hand, the hadron count exceeds the needed number of composites by one when $N_{f}=4$. So a single constraint must exist among $L, M, P$ and $R$ in this case. The precise quantum constraint relation is fixed by symmetries and the weakly coupled $\Lambda_{4} \rightarrow 0$ classical limit. It appears in superpotential form as

$$
W_{N_{f}=4}=X\left[L^{2} \operatorname{det} M+P_{i} M^{i j} P_{j}-R^{2}-L \Lambda_{4}^{10}\right]
$$

where the prefactor $X$ represents a Lagrange multiplier field. As a check on this expression, one can add a mass term for the spinor, integrate out heavy degrees of freedom and flow down to the $S O(7)$ theory with just $N_{f}=4$ vectors. The two separate branches in $N_{f}=N_{c}-3 S O\left(N_{c}\right)$ theory with $W=0$ or $W \propto \Lambda^{b_{0}} / \operatorname{det} M$ are then both properly recovered from $W_{N_{f}=4}$ [3, 17].

The quantum moduli space of degenerate vacua in the $N_{f}=4$ model clearly differs from its classical progenitor as a result of the strong interaction scale $\Lambda_{4}$ appearing on the RHS of (2.7). Yet the origin $L=M=P=R=0$ lies on both. Since the global $S U\left(N_{f}\right) \times U(1)_{Y} \times U(1)_{R}$ symmetry group remains unbroken at this point, it is instructive to compare 't Hooft anomalies in the microscopic and macroscopic theories. We find that the $S U\left(N_{f}\right)^{3}, S U\left(N_{f}\right)^{2} U(1)_{Y}, S U\left(N_{f}\right)^{2} U(1)_{R}, U(1)_{Y}, U(1)_{Y}^{3}, U(1)_{R}, U(1)_{R}^{3}, U(1)_{Y}^{2} U(1)_{R}$ and $U(1)_{R}^{2} U(1)_{Y}$ anomalies all match provided we include a field $X \sim(1 ; 1 ;-8,2)$ into the low energy spectrum which has the same quantum numbers as the $N_{f}=4$ Lagrange multiplier. This nontrivial agreement among nine different 't Hooft anomalies strongly 


\begin{tabular}{|c|c|c|c|c|c|c|}
\hline$N_{f}$ & Hadrons & $L$ & $M$ & $P$ & $R$ & constraints \\
\hline 0 & 1 & 1 & & & & \\
\hline 1 & 2 & 1 & 1 & & & \\
\hline 2 & 4 & 1 & 3 & & & \\
\hline 3 & 8 & 1 & 6 & 1 & & \\
\hline 4 & 15 & 1 & 10 & 4 & 1 & -1 \\
\hline
\end{tabular}

Table 2: Hadron degree of freedom count

suggests that the colorless composites in (2.6) represent the only light degrees of freedom in the low energy theory. It is natural to interpret this finding as evidence for confinement in the $N_{f}=4 S O(7)$ model. On the other hand, the parton and hadron level global anomalies do not match when $N_{f}=5$, and the disagreement cannot be eliminated via inclusion of additional color-singlet fields without disrupting the $N_{f}=4$ results. So we conclude that the $S O(7)$ model ceases to confine at this juncture.

The vacuum structure of the $N_{f}=5$ model is more simply understood in terms of a weakly coupled magnetic dual to the $S O(7)$ electric theory. The dual description has symmetry group

$$
\widetilde{G}=S U\left(N_{f}-3\right)_{\text {local }} \times\left[S U\left(N_{f}\right) \times U(1)_{Y} \times U(1)_{R}\right]_{\text {global }},
$$

superfield matter content

$$
\begin{aligned}
q_{i}^{\alpha} & \sim\left(\square ; \bar{\square} ; \frac{2 N_{f}-3}{N_{f}-3}, \frac{3}{N_{f}} \frac{N_{f}-4}{N_{f}-3}\right) \\
q^{\prime \alpha} & \sim\left(\square ; 1 ; \frac{N_{f}}{N_{f}-3}, \frac{N_{f}-4}{N_{f}-3}\right) \\
s_{\alpha \beta} & \sim\left(\bar{\square} ; 1 ;-\frac{2 N_{f}}{N_{f}-3}, \frac{2}{N_{f}-3}\right) \\
\ell & \sim\left(1 ; 1 ; 2 N_{f}, 0\right) \\
m^{(i j)} & \sim\left(1 ; \square ;-2,2-\frac{8}{N_{f}}\right)
\end{aligned}
$$


and tree level superpotential

$$
W_{\mathrm{mag}}=\mu_{1}^{-2} m^{(i j)} q_{i}^{\alpha} s_{\alpha \beta} q_{j}^{\beta}-q^{\prime \alpha} s_{\alpha \beta} q^{\prime \beta}-\mu_{2}^{4-N_{f}} \ell \operatorname{det} s .
$$

Several points about this magnetic theory should be noted. Firstly, its color gauge group and abelian hypercharge and R-charge global symmetries are nonanomalous like their electric counterparts. Moreover, all nine global 't Hooft anomalies match between the electric and magnetic sides for $N_{f} \geq 4$. This highly nontrivial anomaly agreement provides an important first check on the duality. Secondly, the magnetic Wilsonian beta function coefficient $\widetilde{b_{0}}=2 N_{f}-9$ is positive for $N_{f} \geq 5$. The dual theory is consequently asymptotically free and does not possess a free magnetic phase. Instead, it exists at the origin of moduli space in a nonabelian Coulomb phase for $5 \leq N_{f}<14$ flavors. Thirdly, the dual global symmetry group starts as $\widetilde{G}_{\text {global }}=U\left(N_{f}+1\right)_{q+q^{\prime}} \times U(1)_{s} \times U\left[1+\frac{1}{2} N_{f}\left(N_{f}+1\right)\right]_{\ell+m}$ in the absence of any tree level superpotential. The interaction terms in (2.10) break $\widetilde{G}_{\text {global }}$ down to $S U\left(N_{f}\right) \times U(1)_{Y} \times U(1)_{R}$. The microscopic electric and magnetic theories therefore share the same global symmetry group at short as well as long distance scales. Fourthly, we treat all colored elementary fields in the magnetic theory as canonically normalized. On the other hand, we take the ultraviolet mass dimensions for the color-singlets to equal those of their electric analogues. In order for the magnetic superpotential to have dimension three, its nonrenormalizable interaction terms must be accompanied by appropriate inverse powers of some scales $\mu_{1}$ and $\mu_{2}$. For simplicity, we set these scales equal to unity from here on. Finally, the magnetic theory's matter content belongs to a manifestly chiral representation of $\widetilde{G}$. So this dual to the $S O(7)$ model represents another example of a chiral-nonchiral pair.

In order to check the dual, we look for maps between gauge invariant composites in the electric and magnetic theories. The $L$ and $M^{(i j)}$ hadrons in (2.6) are obviously identified with the elementary $\ell$ and $m^{(i j)}$ fields in (2.9). $P^{[i j k]}$ and $R^{[i j k l]}$ are matched onto the dual quark combinations

$$
\begin{aligned}
& p^{\left[i_{1} i_{2} i_{3}\right]}=\epsilon^{i_{1} \cdots i_{N_{f}}} \epsilon_{\alpha_{1} \cdots \alpha_{N_{f}-3}} q_{i_{4}}^{\alpha_{1}} \cdots q_{i_{N_{f}}}^{\alpha_{N_{f}-3}} \\
& r^{\left[i_{1} \cdots i_{4}\right]}=\epsilon^{i_{1} \cdots i_{N_{f}}} \epsilon_{\alpha_{1} \cdots \alpha_{N_{f}-3}} q_{i_{5}}^{\alpha_{1}} \cdots q_{i_{N_{f}}}^{\alpha_{N_{f}-4}} q^{\prime \alpha_{N_{f}-3}} .
\end{aligned}
$$

These magnetic baryons possess the same global quantum numbers as their electric theory counterparts. Additional gauge invariant operators beyond those relevant for the $S O(7)$ 
model's confining phase can also be mapped. For instance, the electric baryons

$$
\begin{aligned}
B_{0}^{\left[i_{1} \cdots i_{7}\right]} & =\epsilon^{\mu_{1} \cdots \mu_{7}} V_{\mu_{1}}^{i_{1}} \cdots V_{\mu_{7}}^{i_{7}} \\
B_{1}^{a\left[i_{1} \cdots i_{5}\right]} & =\epsilon^{\mu_{1} \cdots \mu_{7}} V_{\mu_{1}}^{i_{1}} \cdots V_{\mu_{5}}^{i_{5}} W_{\mu_{6} \mu_{7}}^{a} \\
B_{2}^{\left[i_{1} i_{2} i_{3}\right]} & =\epsilon^{\mu_{1} \cdots \mu_{7}} V_{\mu_{1}}^{i_{1}} V_{\mu_{2}}^{i_{2}} V_{\mu_{3}}^{i_{3}} W_{\mu_{4} \mu_{5}}^{a} W_{\mu_{6} \mu_{7}}^{a}
\end{aligned}
$$

are identified with magnetic antibaryons built from effective dual antiquarks $\left(\bar{q}_{\text {eff }}\right)_{\alpha i} \equiv$ $s_{\alpha \beta} q_{i}^{\alpha}$ :

$$
\begin{aligned}
b_{0}^{\left[i_{1} \cdots i_{7}\right]} & =\epsilon^{i_{1} \cdots i_{N_{f}}} \epsilon^{\alpha_{1} \cdots \alpha_{N_{f}-3}}(s q)_{\alpha_{1} i_{8}} \cdots(s q)_{\alpha_{N_{f}-7} i_{N_{f}}}\left(s \widetilde{w}^{a}\right)_{\alpha_{N_{f}-6} \alpha_{N_{f}-5}}\left(s \widetilde{w}^{a}\right)_{\alpha_{N_{f}-4} \alpha_{N_{f}-3}} \\
b_{1}^{a\left[i_{1} \cdots i_{5}\right]} & =\epsilon^{i_{1} \cdots i_{N_{f}}} \epsilon^{\alpha_{1} \cdots \alpha_{N_{f}-3}}(s q)_{\alpha_{1} i_{6}} \cdots(s q)_{\alpha_{N_{f}-5} i_{N_{f}}}\left(s \widetilde{w}^{a}\right)_{\alpha_{N_{f}-4} \alpha_{N_{f}-3}} \\
b_{2}^{\left[i_{1} i_{2} i_{3}\right]} & =\epsilon^{i_{1} \cdots i_{N_{f}}} \epsilon^{\alpha_{1} \cdots \alpha_{N_{f}-3}}(s q)_{\alpha_{1} i_{4}} \cdots(s q)_{\alpha_{N_{f}-3} i_{N_{f}}} .
\end{aligned}
$$

The $B_{1}^{a}$ superfield has a simple interpretation in the $N_{f} \geq 5$ electric theory when viewed along flat directions where the $S O(7)$ gauge group is Higgsed down to $S O(2) \simeq U(1)$ : it projects out the abelian Coulomb phase photon from the gluon field strength tensor [1]. Similarly, $B_{2}$ becomes a second glueball in addition to $S=W_{\mu \nu}^{a} W^{\mu \nu a}$ along flat directions where $S O(7)$ breaks to $S O(4) \simeq S U(2) \times S U(2)$.

To further test the duality, we investigate deformations of the electric and magnetic theories which induce renormalization group flows to known dual pairs. We first consider Higgsing $S O(7)$ along the spinor flat direction. When $Q^{A}$ acquires a nonvanishing expectation value, the electric theory's symmetry group breaks down to

$$
H=\left[G_{2}\right]_{\text {local }} \times\left[S U\left(N_{f}\right) \times U(1)_{R}\right]_{\text {global }},
$$

and its 7-dimensional vectors become fundamentals under the $G_{2}$ subgroup:

$$
F_{\alpha}^{i} \sim\left(7 ; \square ; 1-\frac{4}{N_{f}}\right)
$$

The global hypercharge in (2.1) is broken by the spinor vev, but the $U(1)_{R}$ factor remains unaffected since $Q^{A}$ carries zero R-charge. On the magnetic side, we freeze $\ell$ at its induced expectation value and drop the hypercharge assignments for all fields. The dual theory consequently reduces to

$$
\widetilde{H}=S U\left(N_{f}-3\right)_{\text {local }} \times\left[S U\left(N_{f}\right) \times U(1)_{R}\right]_{\text {global }}
$$


with matter content

$$
\begin{aligned}
q_{i}^{\alpha} & \sim\left(\square ; \bar{\square} ; \frac{3}{N_{f}} \frac{N_{f}-4}{N_{f}-3}\right) \\
q^{\prime \alpha} & \sim\left(\square ; 1 ; \frac{N_{f}-4}{N_{f}-3}\right) \\
s_{\alpha \beta} & \sim\left(\bar{\square} ; 1 ; \frac{2}{N_{f}-3}\right) \\
m^{(i j)} & \sim\left(1 ; \square ; 2-\frac{8}{N_{f}}\right)
\end{aligned}
$$

and tree level superpotential

$$
W_{\mathrm{mag}}=m^{(i j)} q_{i}^{\alpha} s_{\alpha \beta} q_{j}^{\beta}-q^{\prime \alpha} s_{\alpha \beta} q^{\prime \beta}-\operatorname{det} s .
$$

This deformation reproduces Pouliot's dual to $G_{2}$ theory with $N_{f}$ fundamentals [16].

We next consider adding a mass term for the spinor field. The $S O(7)$ model then becomes

$$
G=S O(7)_{\text {local }} \times\left[S U\left(N_{f}\right) \times U(1)_{R^{\prime}}\right]_{\text {global }}
$$

with

$$
V_{\mu}^{i} \sim\left(7 ; \square ; 1-\frac{5}{N_{f}}\right) .
$$

In the magnetic theory, we add $W_{\text {tree }}=\mu \ell$ to the superpotential in $(2.10)$ which causes $s_{\alpha \beta}$ to condense. Its expectation value $\left\langle s_{\alpha \beta}\right\rangle$ can always be rotated into diagonal form via an $S U\left(N_{f}-3\right)$ color transformation. But from the $\ell$ field's equation of motion, we learn that $\operatorname{det} s=\mu$ is nonvanishing. We may consequently perform a nonsingular complexified $S U\left(N_{f}-3\right)$ rotation and set each nonzero eigenvalue of $\left\langle s_{\alpha \beta}\right\rangle$ equal to unity. The $s$ field's identity matrix vev then breaks the magnetic gauge group $S U\left(N_{f}-3\right)$ down to $S O\left(N_{f}-3\right)$ and yields a mass term for the $q^{\prime}$ dual quark. After integrating out $q^{\prime}$, we find that the deformed magnetic theory reduces precisely to Seiberg's dual for an $S O(7)$ theory with $N_{f}$ vectors [1, 17].

The most interesting duality result emerges when we give mass to all but four flavors in the electric theory and thereby completely Higgs the magnetic gauge group. Instanton effects generate a new superpotential term beyond those already present in (2.10):

$$
W_{\mathrm{mag}} \stackrel{N_{f} \rightarrow 4}{\longrightarrow} s\left[\ell^{2} \operatorname{det} m+q_{i} m^{(i j)} q_{j}-q^{\prime} q^{\prime}-\ell\right]
$$


Each elementary dual field in this expression can be identified with a corresponding composite hadron in the $N_{f}=4 S O(7)$ theory on the basis of its charge assignments:

$$
\begin{aligned}
q_{i} & \sim(1 ; \bar{\square} ; 5,0) \sim P_{i} \\
q^{\prime} & \sim(1 ; 1 ; 4,0) \sim R \\
s & \sim(1 ; 1 ;-8,2) \sim X \\
\ell & \sim(1 ; 1 ; 8,-8) \sim L \\
m^{(i j)} & \sim(1 ; \square ;-2,2) \sim M^{(i j)} .
\end{aligned}
$$

It is important to note that $s$ has exactly the right quantum numbers to be mapped onto the $X$ Lagrange multiplier. After replacing the magnetic fields in (2.21) by their electric counterparts and restoring dimensionful scales, we reproduce the confining phase $N_{f}=4$ superpotential in (2.7). The quantum constraint among hadrons in the electric theory is thus recovered from semiclassical equations of motion in the magnetic theory. So we see duality at work relating complicated nonperturbative dynamics in one description to weakly coupled phenomena in the other.

\section{3. $S O(7)$ with $N_{f}$ vectors and two spinors}

In this section, we generalize our preceding discussion by adding a second spinor into the $S O(7)$ model. The modified electric theory now has symmetry group

$$
G=S O(7)_{\text {local }} \times\left[S U\left(N_{f}\right) \times S U(2) \times U(1)_{Y} \times U(1)_{R}\right]_{\text {global }},
$$

superfield matter content

$$
\begin{aligned}
V_{\mu}^{i} & \sim\left(7 ; \square, 1 ;-2,1-\frac{5}{N_{f}}\right) \\
Q_{I}^{A} & \sim\left(8 ; 1,2 ; N_{f}, 1\right)
\end{aligned}
$$

and Wilsonian beta function coefficient $b_{0}=13-N_{f}$. The global $S U(2)$ factor which rotates the two spinor fields represents a qualitatively new feature in this model compared to the previous one. We will shortly see how it is implemented in the magnetic dual.

Given the pattern of symmetry breaking realized at generic points in moduli space [21]

$$
S O(7) \stackrel{8}{\longrightarrow} G_{2} \stackrel{8}{\longrightarrow} S U(3) \stackrel{7}{\longrightarrow} S U(2) \stackrel{7}{\longrightarrow} 1
$$


it is straightforward to determine the number of independent gauge invariant operators needed to label flat directions in the second $S O(7)$ model as a function of $N_{f}$. Using the same sorts of counting arguments as in section 2, we find that the hadrons

$$
\begin{aligned}
L_{\mathrm{X}} & =Q_{I}^{T}\left(\sigma_{2} \sigma_{\mathrm{x}}\right)_{I J} C Q_{J} \sim\left(1 ; 1,3 ; 2 N_{f}, 2\right) \\
M^{(i j)} & =\left(V^{T}\right)^{i \mu} V_{\mu}^{j} \sim\left(1 ; \square, 1 ;-4,2-\frac{10}{N_{f}}\right) \\
N^{i} & =Q_{I}^{T}\left(\sigma_{2}\right)_{I J} V^{i} C Q_{J} \sim\left(1 ; \square, 1 ; 2 N_{f}-2,3-\frac{5}{N_{f}}\right) \\
O^{[i j]} & =\frac{1}{2 !} Q_{I}^{T}\left(\sigma_{2}\right)_{I J} V^{[i} V^{j]} C Q_{J} \sim\left(1 ; \text { 日; } 2 N_{f}-4,4-\frac{10}{N_{f}}\right) \\
P_{\mathrm{X}}^{[i j k]} & =\frac{1}{3 !} Q_{I}^{T}\left(\sigma_{2} \sigma_{\mathrm{x}}\right)_{I J} V^{[i} V^{j} V^{k]} C Q_{J} \sim\left(1 ; \text { 日 }, 3 ; 2 N_{f}-6,5-\frac{15}{N_{f}}\right)
\end{aligned}
$$

account for all massless degrees of freedom when $N_{f} \leq 2$. In these operator definitions, $S O(7)$ color, $S U\left(N_{f}\right)$ vector and $S U(2)$ spinor indices are respectively denoted by Greek, small Latin and large Latin letters. We also note that the Pauli matrix factors $\sigma_{2} \sigma_{X}$ and $\sigma_{2}$ combine together spinors into symmetric and antisymmetric products in accord with (2.5).

The hadron count exceeds the required number of composites by two when $N_{f}=3$. So two separate constraints must exist among $L, M, N, O$ and $P$ in this case. These quantum relations are fixed by symmetry considerations and the $\Lambda_{3} \rightarrow 0$ classical limit:

$$
\begin{aligned}
W_{N_{f}=3}=X & {\left[L_{\mathrm{X}} L_{\mathrm{X}} \operatorname{det} M-\frac{1}{2} \epsilon_{i_{1} i_{2} i_{3}} \epsilon_{j_{1} j_{2} j_{3}} M^{i_{1} j_{1}} M^{i_{2} j_{2}} N^{i_{3}} N^{j_{3}}+O_{i} M^{i j} O_{j}-P_{\mathrm{X}} P_{\mathrm{X}}-\Lambda_{3}^{10}\right] } \\
& +Y\left[N^{i} O_{i}-L_{\mathrm{X}} P_{\mathrm{X}}\right] .
\end{aligned}
$$

The first constraint prohibits the point $L=M=N=O=P=0$ from lying on the quantum moduli space. As a result, matching global 't Hooft anomalies at the origin where all global symmetries are unbroken might seem to make little sense. Nevertheless, we find that the parton and hadron level $S U\left(N_{f}\right)^{3}, S U\left(N_{f}\right)^{2} U(1)_{Y}, S U\left(N_{f}\right)^{2} U(1)_{R}, S U(2)^{2} U(1)_{Y}$, $S U(2)^{2} U(1)_{R}, U(1)_{Y}, U(1)_{Y}^{3}, U(1)_{R}, U(1)_{R}^{3}, U(1)_{Y}^{2} U(1)_{R}$ and $U(1)_{R}^{2} U(1)_{Y}$ anomalies all agree when $N_{f}=3$ provided we include the Lagrange multipliers $X \sim(1 ; 1,1 ; 0,2)$ and $Y \sim(1 ; 1,1 ;-6,0)$ into the low energy spectrum. As we shall see, the magnetic dual provides an explanation for this surprising electric theory result.

The dual to the second $S O(7)$ model has symmetry group

$$
\widetilde{G}=S U\left(N_{f}-2\right)_{\text {local }} \times\left[S U\left(N_{f}\right) \times S O(3) \times U(1)_{Y} \times U(1)_{R}\right]_{\text {global }}
$$


matter content

$$
\begin{aligned}
& q_{i}^{\alpha} \sim\left(\square ; \bar{\square}, 1 ; 2, \frac{4 N_{f}-10}{N_{f}\left(N_{f}-2\right)}\right) \\
& q_{\mathrm{x}}^{\prime \alpha} \sim\left(\square ; 1,3 ; 0, \frac{N_{f}-3}{N_{f}-2}\right) \\
& \bar{q}_{\alpha} \sim\left(\bar{\square} ; 1,1 ;-2 N_{f},-\frac{N_{f}-3}{N_{f}-2}\right) \\
& s_{\alpha \beta} \sim\left(\bar{\square} ; 1,1 ; 0, \frac{2}{N_{f}-2}\right) \\
& \ell_{\mathrm{X}} \sim\left(1 ; 1,3 ; 2 N_{f}, 2\right) \\
& m^{(i j)} \sim\left(1 ; \square, 1 ;-4,2-\frac{10}{N_{f}}\right) \\
& n^{i} \sim\left(1 ; \square, 1 ; 2 N_{f}-2,3-\frac{5}{N_{f}}\right)
\end{aligned}
$$

and tree level superpotential

$$
W_{\mathrm{mag}}=\mu_{1}^{-2} n^{i} q_{i}^{\alpha} \bar{q}_{\alpha}-\mu_{2}^{-1} \ell_{\mathrm{x}} q_{\mathrm{x}}^{\alpha} \bar{q}_{\alpha}+\mu_{3}^{-2} m^{(i j)} q_{i}^{\alpha} s_{\alpha \beta} q_{j}^{\beta}-q_{\mathrm{x}}^{\prime \alpha} s_{\alpha \beta}{q_{\mathrm{x}}^{\prime \beta}}^{\beta} \mu_{4}^{5-N_{f}} \operatorname{det} s .
$$

Various points about this dual description should be noted. Firstly, the magnetic gauge group and global hypercharge and R-charge symmetries are nonanomalous, and all global 't Hooft anomalies match between the electric and magnetic theories. Secondly, the magnetic beta function coefficient $\widetilde{b_{0}}=2 N_{f}-8$ is positive for $N_{f} \geq 5$. When $N_{f}=4$, the last determinant term in $W_{\text {mag }}$ reduces to a quadratic mass term for $s_{\alpha \beta}$. After removing this field from the low energy effective theory, we find $\widetilde{b_{0}}=2$. So the magnetic theory is asymptotically free and exists at the moduli space origin in a nonabelian Coulomb phase for $4 \leq N_{f}<13$. Finally, the magnetic superpotential interactions break the initial $U\left(N_{f}+3\right)_{q+q^{\prime}} \times U(1)_{\bar{q}} \times U(1)_{s} \times U\left[3+N_{f}+\frac{1}{2} N_{f}\left(N_{f}+1\right)\right]_{\ell+m+n}$ global symmetry down to $\widetilde{G}_{\text {global }}=S U\left(N_{f}\right) \times S O(3) \times U(1) \times U(1)$. We again set the various dimensionful scales that appear in $W_{\text {mag }}$ to unity for simplicity.

Mappings between electric and magnetic gauge invariant composites in this second $S O(7)$ model are straightforward generalizations of those in the first. The $L_{\mathrm{x}}, M^{(i j)}$ and $N^{i}$ hadrons in (3.4) are identified with their $\ell_{\mathrm{X}}, m^{(i j)}$ and $n^{i}$ counterparts in (3.7), while $O^{[i j]}$ and $P_{\mathrm{X}}^{[i j k]}$ are matched onto

$$
\begin{aligned}
o^{\left[i_{1} i_{2}\right]} & =\epsilon^{i_{1} \cdots i_{N_{f}}} \epsilon_{\alpha_{1} \cdots \alpha_{N_{f}-2}} q_{i_{3}}^{\alpha_{1}} \cdots q_{i_{N_{f}}}^{\alpha_{N_{f}-2}} \\
p_{\mathrm{X}}^{\left[i_{1} i_{2} i_{3}\right]} & =\epsilon^{i_{1} \cdots i_{N_{f}}} \epsilon_{\alpha_{1} \cdots \alpha_{N_{f}-2}} q_{i_{4}}^{\alpha_{1}} \cdots q_{i_{N_{f}}}^{\alpha_{N_{f}-3}} q^{\prime \alpha^{\alpha_{N_{f}-2}}} .
\end{aligned}
$$


The electric baryons

$$
\begin{aligned}
B_{0}^{\left[i_{1} \cdots i_{7}\right]} & =\epsilon^{\mu_{1} \cdots \mu_{7}} V_{\mu_{1}}^{i_{1}} \cdots V_{\mu_{7}}^{i_{7}} \\
B_{1}^{a\left[i_{1} \cdots i_{5}\right]} & =\epsilon^{\mu_{1} \cdots \mu_{7}} V_{\mu_{1}}^{i_{1}} \cdots V_{\mu_{5}}^{i_{5}} W_{\mu_{6} \mu_{7}}^{a} \\
B_{2}^{\left[i_{1} i_{2} i_{3}\right]} & =\epsilon^{\mu_{1} \cdots \mu_{7}} V_{\mu_{1}}^{i_{1}} V_{\mu_{2}}^{i_{2}} V_{\mu_{3}}^{i_{3}} W_{\mu_{4} \mu_{5}}^{a} W_{\mu_{6} \mu_{7}}^{a}
\end{aligned}
$$

are identified with the magnetic composites

$$
\begin{aligned}
b_{0}^{\left[i_{1} \cdots i_{7}\right]} & =\epsilon^{i_{1} \cdots i_{N_{f}}} \epsilon^{\alpha_{1} \cdots \alpha_{N_{f}-2}}(s q)_{\alpha_{1} i_{8}} \cdots(s q)_{\alpha_{N_{f}-7} i_{N_{f}}}\left(s \widetilde{w}^{a}\right)_{\alpha_{N_{f}-6} \alpha_{N_{f}-5}}\left(s \widetilde{w}^{a}\right)_{\alpha_{N_{f}-4} \alpha_{N_{f}-3}} \bar{q}_{\alpha_{N_{f}-2}} \\
b_{1}^{a\left[i_{1} \cdots i_{5}\right]} & =\epsilon^{i_{1} \cdots i_{N_{f}}} \epsilon^{\alpha_{1} \cdots \alpha_{N_{f}-2}}(s q)_{\alpha_{1} i_{6}} \cdots(s q)_{\alpha_{N_{f}-5} i_{N_{f}}}\left(s \widetilde{w}^{a}\right)_{\alpha_{N_{f}-4} \alpha_{N_{f}-3}} \bar{q}_{\alpha_{N_{f}-2}} \\
b_{2}^{\left[i_{1} i_{2} i_{3}\right]} & =\epsilon^{i_{1} \cdots i_{N_{f}}} \epsilon^{\alpha_{1} \cdots \alpha_{N_{f}-2}}(s q)_{\alpha_{1} i_{4}} \cdots(s q)_{\alpha_{N_{f}-3} i_{N_{f}}} \bar{q}_{\alpha_{N_{f}-2}} .
\end{aligned}
$$

We can also form more exotic combinations of vectors, spinors and gluons

$$
\begin{aligned}
C_{0}^{\left[i_{1} \cdots i_{6}\right]} & =\epsilon^{\mu_{1} \cdots \mu_{7}} V_{\mu_{1}}^{i_{1}} \cdots V_{\mu_{6}}^{i_{6}} Q_{I}^{T}\left(\sigma_{2}\right)_{I J} \Gamma_{\mu_{7}} C Q_{J} \\
C_{1}^{a\left[i_{1} \cdots i_{4}\right]} & =\epsilon^{\mu_{1} \cdots \mu_{7}} V_{\mu_{1}}^{i_{1}} \cdots V_{\mu_{4}}^{i_{4}} W_{\mu_{5} \mu_{6}}^{a} Q_{I}^{T}\left(\sigma_{2}\right)_{I J} \Gamma_{\mu_{7}} C Q_{J} \\
C_{2}^{\left[i_{1} i_{2}\right]} & =\epsilon^{\mu_{1} \cdots \mu_{7}} V_{\mu_{1}}^{i_{1}} V_{\mu_{2}}^{i_{2}} W_{\mu_{3} \mu_{4}}^{a} W_{\mu_{5} \mu_{6}}^{a} Q_{I}^{T}\left(\sigma_{2}\right)_{I J} \Gamma_{\mu_{7}} C Q_{J}
\end{aligned}
$$

which map onto the dual antibaryons

$$
\begin{aligned}
c_{0}^{\left[i_{1} \cdots i_{6}\right]} & =\epsilon^{i_{1} \cdots i_{N_{f}}} \epsilon^{\alpha_{1} \cdots \alpha_{N_{f}-2}}(s q)_{\alpha_{1} i_{7}} \cdots(s q)_{\alpha_{N_{f}-6} i_{N_{f}}}\left(s \widetilde{w}^{a}\right)_{\alpha_{N_{f}-5} \alpha_{N_{f}-4}}\left(s \widetilde{w}^{a}\right)_{\alpha_{N_{f}-3} \alpha_{N_{f}-2}} \\
c_{1}^{a\left[i_{1} \cdots i_{4}\right]} & =\epsilon^{i_{1} \cdots i_{N_{f}}} \epsilon^{\alpha_{1} \cdots \alpha_{N_{f}-2}}(s q)_{\alpha_{1} i_{5}} \cdots(s q)_{\alpha_{N_{f}-4} i_{N_{f}}}\left(s \widetilde{w}^{a}\right)_{\alpha_{N_{f}-3} \alpha_{N_{f}-2}} \\
c_{2}^{\left[i_{1} i_{2}\right]} & =\epsilon^{i_{1} \cdots i_{N_{f}}} \epsilon^{\alpha_{1} \cdots \alpha_{N_{f}-2}}(s q)_{\alpha_{1} i_{3}} \cdots(s q)_{\alpha_{N_{f}-2} i_{N_{f}}} .
\end{aligned}
$$

The consistency of all these operator identifications provides strong support for the duality hypothesis.

The long distance equivalence between the microscopic $S O(7)$ and $S U\left(N_{f}-2\right)$ theories can be probed via deformations along various flat directions. Rather than repeat the same checks as described in section 2, we focus instead upon recovering our first model from the second. We start by adding the spinor mass term $W_{\text {tree }}=\mu Q_{2}^{T} C Q_{2} \simeq-\frac{1}{2} \mu\left(\ell_{1}+i \ell_{2}\right)$ to both the electric and magnetic theories. In the former, we simply integrate out $Q_{2}$ and flow down to the $S O(7)$ model with just one spinor. In the latter, we eliminate the fields $\ell_{1}, \ell_{2}$ and $n^{i}$ which grow heavy since their electric counterparts contain the second spinor. 
Their equations of motion force the condensates

$$
\begin{aligned}
\left\langle q_{1}^{\prime \alpha}\right\rangle & =\left(0, \cdots, 0, \sqrt{\frac{\mu}{2}}\right) \\
\left\langle q_{2}^{\prime \alpha}\right\rangle & =\left(0, \cdots, 0, i \sqrt{\frac{\mu}{2}}\right) \\
\left\langle\bar{q}_{\alpha}\right\rangle & =\left(0, \cdots, 0, \sqrt{\frac{\mu}{2}}\right) \\
\left\langle q_{i}^{N_{f}-2}\right\rangle & =0
\end{aligned}
$$

to develop which break the $S U\left(N_{f}-2\right)$ gauge group down to $S U\left(N_{f}-3\right)$. After these vevs are substituted back into $W_{\text {mag }}$ and the fields $\ell_{3}$ and $q_{3}^{\prime N_{f}-2}$ that acquire induced masses are integrated out, the superpotential reduces to

$$
W_{\mathrm{mag}} \rightarrow m^{(i j)} \hat{q}_{i}^{\alpha} \hat{s}_{\alpha \beta} \hat{q}_{j}^{\beta}-\hat{q}_{3}^{\alpha} \hat{s}_{\alpha \beta} \hat{q}_{3}^{\beta}-\left(s_{N_{f}-2 N_{f}-2}\right) \operatorname{det} \hat{s}
$$

where all hatted objects transform under $S U\left(N_{f}-3\right)$. This expression reproduces the superpotential in (2.10) when all hats are dropped and the fields $\hat{q}_{3}^{\alpha} \rightarrow q^{\prime a}$ and $s_{N_{f}-2 N_{f}-2} \rightarrow \ell$ are renamed. We thus recover the dual to our first $S O(7)$ model.

It is once again instructive to investigate the changes that arise in the dual pair when the number of vector flavors in the electric theory is reduced down to $N_{f}=3$ and the magnetic gauge group becomes totally Higgsed. In this case, the superpotential in (3.8) reduces to

$$
\begin{gathered}
W_{\mathrm{mag}} \stackrel{N_{f} \rightarrow 3}{\longrightarrow} s\left[\ell_{\mathrm{x}} \ell_{\mathrm{x}} \operatorname{det} m-\frac{1}{2} \epsilon_{i_{1} i_{2} i_{3}} \epsilon_{j_{1} j_{2} j_{3}} m^{i_{1} j_{1}} m^{i_{2} j_{2}} n^{i_{3} j_{3}}+q_{i} m^{(i j)} q_{j}-q_{\mathrm{x}}^{\prime} q_{\mathrm{X}}^{\prime}-1\right] \\
+\bar{q}\left[n^{i} q_{i}-\ell_{\mathrm{x}} q_{\mathrm{x}}^{\prime}\right] .
\end{gathered}
$$

We have added by hand the first two terms on the RHS even though we have not identified their physical origin. Consistency with confining phase results requires that these terms be generated. After identifying all the dual fields in (3.16) with confined electric composites on the basis of their charge assignments

$$
\begin{aligned}
q_{i} & \sim\left(1 ; \bar{\square}, 1 ; 2, \frac{2}{3}\right) \sim O_{i} \\
q_{\mathrm{X}}^{\prime} & \sim(1 ; 1,3 ; 0,0) \sim P_{\mathrm{X}} \\
\bar{q} & \sim(1 ; 1,1 ;-6,0) \sim Y \\
s & \sim(1 ; 1,1 ; 0,2) \sim X \\
\ell_{\mathrm{X}} & \sim(1 ; 1,3 ; 6,2) \sim L_{\mathrm{X}} \\
m^{(i j)} & \sim\left(1 ; \square, 1 ;-4, \frac{4}{3}\right) \sim M^{(i j)} \\
n^{i} & \sim\left(1 ; \square, 1 ; 4, \frac{1}{3}\right) \sim N^{i},
\end{aligned}
$$


we recover the $N_{f}=3$ superpotential in (3.5). It is important to note that $s$ and $\bar{q}$ have exactly the same quantum numbers as the $X$ and $Y$ Lagrange multipliers. So we now understand the origin of these multiplier fields in the low energy effective theory. We also observe that the general agreement between electric and magnetic global anomalies which continues to hold even when the dual gauge group is completely Higgsed in conjunction with the field identifications in (3.17) ensures parton and hadron level anomaly matching in the $N_{f}=3$ model. So as advertised, duality sheds light upon the 't Hooft anomaly agreement.

\section{4. $S O(8)$ with $N_{f}$ vectors, one spinor and one conjugate spinor}

The electric theory in the third chiral-nonchiral dual pair which we shall investigate has symmetry group

$$
G=S O(8)_{\text {local }} \times\left[S U\left(N_{f}\right) \times U(1)_{Y} \times U(1)_{B} \times U(1)_{R}\right]_{\text {global }},
$$

matter superfields

$$
\begin{aligned}
V_{\mu}^{i} & \sim\left(8_{V} ; \square ;-2,0,1-\frac{6}{N_{f}}\right) \\
Q_{S}^{A} & \sim\left(8_{S} ; 1 ; N_{f}, 1,1\right) \\
Q_{C}^{\dot{A}} & \sim\left(8_{C} ; 1 ; N_{f},-1,1\right)
\end{aligned}
$$

and Wilsonian beta function coefficient $b_{0}=16-N_{f}$. The discrete triality transformation that shuffles the vector, spinor and conjugate spinor representations among each other relates this model to two others with different 8-dimensional irrep assignments. As the structure of the $S O(8)$ theory and its dual are similar to the preceding $S O(7)$ models, we will only briefly sketch their basic outlines in this section.

Simple counting arguments demonstrate that the composite operators

$$
\begin{aligned}
L_{S} & =Q_{S}^{T} C Q_{S} \sim\left(1 ; 1 ; 2 N_{f}, 2,2\right) \\
L_{C} & =Q_{C}^{T} C Q_{C} \sim\left(1 ; 1 ; 2 N_{f},-2,2\right) \\
M^{(i j)} & =\left(V^{T}\right)^{i \mu} V_{\mu}^{j} \sim\left(1 ; \square ;-4,0,2-\frac{12}{N_{f}}\right) \\
N^{i} & =Q_{S}^{T} Y^{i} C Q_{C} \sim\left(1 ; \text { 日 } ; 2 N_{f}-2,0,3-\frac{6}{N_{f}}\right) \\
P^{[i j k]} & =\frac{1}{3 !} Q_{S}^{T} Y^{[i} Y^{j} Y^{k]} C Q_{C} \sim\left(1 ; \text { 日; } 2 N_{f}-6,0,5-\frac{18}{N_{f}}\right) \\
R_{S}^{[i j k l]} & =\frac{1}{4 !} Q_{S}^{T} Y^{[i} Y^{j} Y^{k} Y^{l]} C Q_{S} \sim\left(1 ; \text { 目; } 2 N_{f}-8,2,6-\frac{24}{N_{f}}\right) \\
R_{C}^{[i j k l]} & =\frac{1}{4 !} Q_{C}^{T} Y^{[i} Y^{j} Y^{k} Y^{l]} C Q_{C} \sim\left(1 ; \text { 目; } ; 2 N_{f}-8,-2,6-\frac{24}{N_{f}}\right)
\end{aligned}
$$


account for all massless degrees of freedom in the $S O(8)$ model with $N_{f} \leq 3$ vector flavors. In these operator definitions, we treat the spinor and conjugate spinor fields as the projections $Q_{S}=P_{+} Q$ and $Q_{C}=P_{-} Q$ where $Q$ denotes a 16-dimensional spinor of $S O(9)$ and $P_{ \pm}=\frac{1}{2}\left(1 \pm \Gamma_{9}\right)$. The $16 \times 16$ Gamma matrices hidden inside $Y^{i}=\sum_{i=1}^{8} V_{\mu}^{i} \Gamma^{\mu}$ along with the charge conjugation matrix $C$ therefore originate from the Clifford algebra for $S O(9)$.

The same degree of freedom counting indicates that the hadrons in (4.3) are not all independent when $N_{f}=4$. They are instead restricted by the two quantum constraints displayed below in superpotential form:

$$
\begin{aligned}
W_{N_{f}=4}=X & {\left[L_{S} L_{C} \operatorname{det} M-\frac{1}{3 !} \epsilon_{i_{1} i_{2} i_{3} i_{4}} \epsilon_{j_{1} j_{2} j_{3} j_{4}} M^{i_{1} j_{1}} M^{i_{2} j_{2}} M^{i_{3} j_{3}} N^{i_{4}} N^{j_{4}}-P_{i} M^{i j} P_{j}\right.} \\
& \left.-R_{S} R_{C}-\Lambda_{4}^{12}\right]+Y\left[2 N^{i} P_{i}+L_{S} R_{C}-L_{C} R_{S}\right] .
\end{aligned}
$$

Checking global anomalies at the parton and hadron levels, we once again find that they all match in the $N_{f}=4$ theory provided the Lagrange multipliers $X \sim(1 ; 1 ; 0,0,2)$ and $Y \sim(1 ; 1 ;-8,0,0)$ are included into the effective theory's spectrum.

The magnetic dual to the $S O(8)$ model has the symmetry group

$$
\widetilde{G}=S U\left(N_{f}-3\right)_{\text {local }} \times\left[S U\left(N_{f}\right) \times U(1)_{Y} \times U(1)_{B} \times U(1)_{R}\right]_{\text {global }}
$$

and the rather complicated matter content

$$
\begin{aligned}
q_{i}^{\alpha} & \sim\left(\square ; \bar{\square} ; 2,0, \frac{5 N_{f}-18}{N_{f}\left(N_{f}-3\right)}\right) \\
{q^{\prime \alpha}}^{\alpha} & \sim\left(\square ; 1 ; 0,2, \frac{N_{f}-4}{N_{f}-3}\right) \\
{q^{\prime \prime}}^{\alpha} & \sim\left(\square ; 1 ; 0,-2, \frac{N_{f}-4}{N_{f}-3}\right) \\
\bar{q}_{\alpha} & \sim\left(\bar{\square} ; 1 ;-2 N_{f}, 0,-\frac{N_{f}-4}{N_{f}-3}\right) \\
s_{\alpha \beta} & \sim\left(\bar{\square} ; 1 ; 0,0, \frac{2}{N_{f}-3}\right) \\
\ell_{S} & \sim\left(1 ; 1 ; 2 N_{f}, 2,2\right) \\
\ell_{C} & \sim\left(1 ; 1 ; 2 N_{f},-2,2\right) \\
m^{(i j)} & \sim\left(1 ; \square ;-4,0,2-\frac{12}{N_{f}}\right) \\
n^{i} & \sim\left(1 ; \square ; 2 N_{f}-2,0,3-\frac{6}{N_{f}}\right) .
\end{aligned}
$$


Its tree level superpotential looks like

$$
W_{\mathrm{mag}}=2 n^{i} q_{i}^{\alpha} \bar{q}_{\alpha}+\ell_{S} q^{\prime \prime \alpha} \bar{q}_{\alpha}-\ell_{C} q^{\prime \alpha} \bar{q}_{\alpha}-m^{(i j)} q_{i}^{\alpha} s_{\alpha \beta} q_{j}^{\beta}-q^{\prime \alpha} s_{\alpha \beta} q^{\prime \prime \beta}-\operatorname{det} s
$$

once we set to unity all dimensionful scales which multiply nonrenormalizable interaction terms. It is straightforward to verify that the $S U\left(N_{f}\right)^{3}, S U\left(N_{f}\right)^{2} U(1)_{Y}, S U\left(N_{f}\right)^{2} U(1)_{B}$, $S U\left(N_{f}\right)^{2} U(1)_{R}, U(1)_{Y}, U(1)_{Y}^{3}, U(1)_{B}, U(1)_{B}^{3}, U(1)_{R}, U(1)_{R}^{3}, U(1)_{Y}^{2} U(1)_{B}, U(1)_{Y}^{2} U(1)_{R}$, $U(1)_{B}^{2} U(1)_{Y}, U(1)_{B}^{2} U(1)_{R}, U(1)_{R}^{2} U(1)_{Y}$ and $U(1)_{R}^{2} U(1)_{B}$ global anomalies match between the electric and magnetic theories. We also observe that the dual beta function coefficient

$$
\widetilde{b_{0}}= \begin{cases}2, & N_{f}=5 \\ 2 N_{f}-10, & N_{f} \geq 6\end{cases}
$$

is positive everywhere throughout the magnetic theory's range of validity. The dual pair consequently exists in a nonabelian Coulomb phase for $5 \leq N_{f}<16$.

Operator maps between electric and magnetic gauge invariant operators are similar to those in the previous two $S O(7)$ models. $L_{S}, L_{C}, M^{(i j)}$ and $N^{i}$ in (4.3) are mapped onto $\ell_{S}, \ell_{C}, m^{(i j)}$ and $n^{i}$ in (4.6), while $P^{[i j k]}, R_{S}^{[i j k l]}$ and $R_{C}^{[i j k l]}$ are identified with the magnetic baryons

$$
\begin{aligned}
& p^{\left[i_{1} i_{2} i_{3}\right]}=\epsilon^{i_{1} \cdots i_{N_{f}}} \epsilon_{\alpha_{1} \cdots \alpha_{N_{f}-3}} q_{i_{4}}^{\alpha_{1}} \cdots q_{i_{N_{f}}}^{\alpha_{N_{f}-3}} \\
& r_{S}^{\left[i_{1} \cdots i_{4}\right]}=\epsilon^{i_{1} \cdots i_{N_{f}}} \epsilon_{\alpha_{1} \cdots \alpha_{N_{f}-3}} q_{i_{5}}^{\alpha_{1}} \cdots q_{i_{N_{f}}}^{\alpha_{N_{f}-4}} q^{\prime \alpha_{N_{f}-3}} \\
& r_{C}^{\left[i_{1} \cdots i_{4}\right]}=\epsilon^{i_{1} \cdots i_{N_{f}}} \epsilon_{\alpha_{1} \cdots \alpha_{N_{f}-3}} q_{i_{5}}^{\alpha_{1}} \cdots q_{i_{N_{f}}}^{\alpha_{N_{f}-4}} q^{\prime \prime \alpha_{N_{f}-3}} .
\end{aligned}
$$

The electric baryons

$$
\begin{aligned}
B_{0}^{\left[i_{1} \cdots i_{8}\right]} & =\epsilon^{\mu_{1} \cdots \mu_{8}} V_{\mu_{1}}^{i_{1}} \cdots V_{\mu_{8}}^{i_{8}} \\
B_{1}^{a\left[i_{1} \cdots i_{6}\right]} & =\epsilon^{\mu_{1} \cdots \mu_{8}} V_{\mu_{1}}^{i_{1}} \cdots V_{\mu_{6}}^{i_{6}} W_{\mu_{7} \mu_{8}}^{a} \\
B_{2}^{\left[i_{1} \cdots i_{4}\right]} & =\epsilon^{\mu_{1} \cdots \mu_{8}} V_{\mu_{1}}^{i_{1}} \cdots V_{\mu_{4}}^{i_{4}} W_{\mu_{5} \mu_{6}}^{a} W_{\mu_{7} \mu_{8}}^{a}
\end{aligned}
$$

are matched onto the magnetic composites

$$
\begin{aligned}
b_{0}^{\left[i_{1} \cdots i_{8}\right]} & =\epsilon^{i_{1} \cdots i_{N_{f}}} \epsilon^{\alpha_{1} \cdots \alpha_{N_{f}-3}}(s q)_{\alpha_{1} i_{9}} \cdots(s q)_{\alpha_{N_{f}-8} i_{N_{f}}}\left(s \widetilde{w}^{a}\right)_{\alpha_{N_{f}-7} \alpha_{N_{f}-6}}\left(s \widetilde{w}^{a}\right)_{\alpha_{N_{f}-5} \alpha_{N_{f}-4}} \bar{q}_{\alpha_{N_{f}-3}} \\
b_{1}^{a\left[i_{1} \cdots i_{6}\right]} & =\epsilon^{i_{1} \cdots i_{N_{f}}} \epsilon^{\alpha_{1} \cdots \alpha_{N_{f}-3}}(s q)_{\alpha_{1} i_{7}} \cdots(s q)_{\alpha_{N_{f}-6} i_{N_{f}}}\left(s \widetilde{w}^{a}\right)_{\alpha_{N_{f}-5} \alpha_{N_{f}-4}} \bar{q}_{\alpha_{N_{f}-3}} \\
b_{2}^{\left[i_{1} \cdots i_{4}\right]} & =\epsilon^{i_{1} \cdots i_{N_{f}}} \epsilon^{\alpha_{1} \cdots \alpha_{N_{f}-3}}(s q)_{\alpha_{1} i_{5}} \cdots(s q)_{\alpha_{N_{f}-4} i_{N_{f}}} \bar{q}_{\alpha_{N_{f}-3}} .
\end{aligned}
$$


Finally, the exotic $S O(8)$ combinations of spinor, vector and gluon superfields

$$
\begin{aligned}
C_{0}^{\left[i_{1} \cdots i_{7}\right]} & =\epsilon^{\mu_{1} \cdots \mu_{8}} V_{\mu_{1}}^{i_{1}} \cdots V_{\mu_{7}}^{i_{7}} Q_{S}^{T} \Gamma_{\mu_{8}} C Q_{C} \\
C_{1}^{a\left[i_{1} \cdots i_{5}\right]} & =\epsilon^{\mu_{1} \cdots \mu_{8}} V_{\mu_{1}}^{i_{1}} \cdots V_{\mu_{5}}^{i_{5}} W_{\mu_{6} \mu_{7}}^{a} Q_{S}^{T} \Gamma_{\mu_{8}} C Q_{C} \\
C_{2}^{\left[i_{1} i_{2} i_{3}\right]} & =\epsilon^{\mu_{1} \cdots \mu_{8}} V_{\mu_{1}}^{i_{1}} V_{\mu_{2}}^{i_{2}} V_{\mu_{3}}^{i_{3}} W_{\mu_{4} \mu_{5}}^{a} W_{\mu_{6} \mu_{7}}^{a} Q_{S}^{T} \Gamma_{\mu_{8}} C Q_{C}
\end{aligned}
$$

are associated with their $S U\left(N_{f}-3\right)$ counterparts

$$
\begin{aligned}
& c_{0}^{\left[i_{1} \cdots i_{7}\right]}=\epsilon^{i_{1} \cdots i_{N_{f}}} \epsilon^{\alpha_{1} \cdots \alpha_{N_{f}-3}}(s q)_{\alpha_{1} i_{8}} \cdots(s q)_{\alpha_{N_{f}-7} i_{N_{f}}}\left(s \widetilde{w}^{a}\right)_{\alpha_{N_{f}-6} \alpha_{N_{f}-5}}\left(s \widetilde{w}^{a}\right)_{\alpha_{N_{f}-4} \alpha_{N_{f}-3}} \\
& c_{1}^{a\left[i_{1} \cdots i_{5}\right]}=\epsilon^{i_{1} \cdots i_{N_{f}}} \epsilon^{\alpha_{1} \cdots \alpha_{N_{f}-3}}(s q)_{\alpha_{1} i_{6}} \cdots(s q)_{\alpha_{N_{f}-5} i_{N_{f}}}\left(s \widetilde{w}^{a}\right)_{\alpha_{N_{f}-4} \alpha_{N_{f}-3}} \\
& c_{2}^{\left[i_{1} i_{2} i_{3}\right]}=\epsilon^{i_{1} \cdots i_{N_{f}}} \epsilon^{\alpha_{1} \cdots \alpha_{N_{f}-3}}(s q)_{\alpha_{1} i_{4}} \cdots(s q)_{\alpha_{N_{f}-3} i_{N_{f}}} .
\end{aligned}
$$

Renormalization group flows induced by various deformations can be used to test the duality relationship between the $S O(8)$ and $S U\left(N_{f}-3\right)$ models. For example, we can integrate out all but four vector flavors. The two quantum constraints encoded within the confining phase superpotential in (4.4) are then recovered from the magnetic theory via semiclassical equations of motion in exactly the same fashion as we have seen in sections 2 and 3. Here we will just consider adding a tree level mass term for the conjugate spinor field. The electric theory then reduces to the Pouliot-Strassler $S O(8)$ model with $N_{f}$ vectors and one spinor after the heavy field is integrated out [17]. On the magnetic side, we supplement the superpotential in (4.7) with $W_{\text {tree }}=\mu \ell_{C}$ and eliminate all fields involving the conjugate spinor. From the equations of motion for $\ell_{C}$ and $n^{i}$, we learn that the condensates

$$
\begin{aligned}
\left\langle q^{\prime \alpha}\right\rangle & =(0, \cdots, 0, \sqrt{\mu}) \\
\left\langle\bar{q}_{\alpha}\right\rangle & =(0, \cdots, 0, \sqrt{\mu}) \\
\left\langle q_{i}^{N_{f}-3}\right\rangle & =0
\end{aligned}
$$

break the magnetic $S U\left(N_{f}-3\right)$ gauge group down to $S U\left(N_{f}-4\right)$. They also generate a bilinear mass term for $q^{\prime \prime N_{f}-3}$ and $\ell_{S}$. After some algebraic manipulations, we find

$$
W_{\mathrm{mag}} \rightarrow-m^{(i j)} \hat{q}_{i}^{\alpha} \hat{s}_{\alpha \beta} \hat{q}_{j}^{\beta}-\ell_{S} \operatorname{det} \hat{s}
$$

which is consistent with the magnetic superpotential expression in ref. [17]. 


\section{5. $S O(9)$ with $N_{f}$ vectors and one spinor}

The final orthogonal group supersymmetric gauge theory which we investigate is based upon

$$
G=S O(9)_{\text {local }} \times\left[S U\left(N_{f}\right) \times U(1)_{Y} \times U(1)_{R}\right]_{\text {global }}
$$

and contains the matter fields

$$
\begin{aligned}
V_{\mu}^{i} & \sim\left(9 ; \square ;-2,1-\frac{5}{N_{f}}\right) \\
Q^{A} & \sim\left(16 ; 1 ; N_{f}, 0\right) .
\end{aligned}
$$

As both the 9-dimensional vector and 16-dimensional spinor irreps of $S O(9)$ are real, this model is nonchiral. It is also asymptotically free provided it contains $N_{f}<19$ vector flavors so that its Wilsonian beta function coefficient $b_{0}=19-N_{f}$ is positive and its full beta function is negative.

At generic points in moduli space, the model follows the gauge symmetry breaking pattern 21

$$
S O(9) \stackrel{16}{\longrightarrow} S O(7) \stackrel{9}{\longrightarrow} G_{2} \stackrel{9}{\longrightarrow} S U(3) \stackrel{9}{\longrightarrow} S U(2) \stackrel{9}{\longrightarrow} 1 .
$$

The first link in this chain indicates that the color group breaks to $S O(7)$ along the spinor flat direction. As we shall see, the electric $S O(9)$ theory and its magnetic counterpart reduce to Pouliot's original chiral-nonchiral dual pair along this direction [18]. Taking into account the symmetry breaking information in (5.3) as well as the spinor tensor product decomposition

$$
16 \times 16=[0]_{S}+[1]_{S}+[2]_{A}+[3]_{A}+[4]_{S},
$$

we form the composite operators

$$
\begin{aligned}
L & =Q^{T} C Q \sim\left(1 ; 1 ; 2 N_{f}, 0\right) \\
M^{(i j)} & =\left(V^{T}\right)^{i \mu} V_{\mu}^{j} \sim\left(1 ; \square ;-4,2-\frac{10}{N_{f}}\right) \\
N^{i} & =Q^{T} Y^{i} C Q \sim\left(1 ; \square ; 2 N_{f}-2,1-\frac{5}{N_{f}}\right) \\
R^{[i j k l]} & =\frac{1}{4 !} Q^{T} Y^{[i} Y^{j} V^{k} Y^{l]} C Q \sim\left(1 ; \text { 日 } ; 2 N_{f}-8 ; 4-\frac{20}{N_{f}}\right) \\
T^{[i j k l m]} & =\frac{1}{5 !} Q^{T} Y^{[i} Y^{j} V^{k} Y^{l} Y^{m]} C Q \sim\left(1 ; \text { 日 } ; 2 N_{f}-10 ; 5-\frac{25}{N_{f}}\right) .
\end{aligned}
$$


Simple degree of freedom counting demonstrates that these hadrons represent all massless modes in the $N_{f} \leq 4$ models. On the other hand, the hadron count exceeds the number of independent scalar potential flat directions by two when $N_{f}=5$. The operators in (5.5) are then constrained by the two quantum relations contained within

$$
\begin{aligned}
W_{N_{f}=5}=X & {\left[L^{2} \operatorname{det} M-\frac{1}{4 !} \epsilon_{i_{1} i_{2} i_{3} i_{4} i_{5}} \epsilon_{j_{1} j_{2} j_{3} j_{4} j_{5}} M^{i_{1} j_{1}} M^{i_{2} j_{2}} M^{i_{3} j_{3}} M^{i_{4} j_{4}} N^{i_{5}} N^{j_{5}}\right.} \\
& \left.-R_{i} M^{i j} R_{j}+T^{2}-\Lambda_{5}^{14}\right]+Y\left[N^{i} R_{i}-L T\right] .
\end{aligned}
$$

In order to check the validity of these two $N_{f}=5$ quantum constraints, it is instructive to determine how they decompose along the spinor flat direction. To begin, we need to explicitly embed the $S O(7)$ subgroup inside $S O(9)$. Working with the $16 \times 16$ Gamma matrices

$$
\begin{array}{lll}
\Gamma_{1}=\sigma_{2} \times \sigma_{3} \times \sigma_{3} \times \sigma_{3} & \Gamma_{4}=-1 \times \sigma_{1} \times \sigma_{3} \times \sigma_{3} & \Gamma_{7}=1 \times 1 \times 1 \times \sigma_{2} \\
\Gamma_{2}=-\sigma_{1} \times \sigma_{3} \times \sigma_{3} \times \sigma_{3} & \Gamma_{5}=1 \times 1 \times \sigma_{2} \times \sigma_{3} & \Gamma_{8}=-1 \times 1 \times 1 \times \sigma_{1} \\
\Gamma_{3}=1 \times \sigma_{2} \times \sigma_{3} \times \sigma_{3} & \Gamma_{6}=-1 \times 1 \times \sigma_{1} \times \sigma_{3} & \Gamma_{9}=\sigma_{3} \times \sigma_{3} \times \sigma_{3} \times \sigma_{3},
\end{array}
$$

we first form the spinor irrep generators $M_{\mu \nu}=-\frac{i}{4}\left[\Gamma_{\mu}, \Gamma_{\nu}\right]$ and then take $M_{12} \equiv H_{1}$, $M_{34} \equiv H_{2}, M_{56} \equiv H_{3}$ and $M_{78} \equiv H_{4}$ as the four members of the $S O(9)$ Cartan subalgebra. We next act upon $Q$ with

$$
\begin{aligned}
& h_{1}=H_{1}+H_{2}-H_{3}-H_{4} \\
& h_{2}=H_{1}-H_{2}-H_{3}+H_{4} \\
& h_{3}=H_{1}-H_{2}+H_{3}-H_{4}
\end{aligned}
$$

and find that the flat direction vev $\langle Q\rangle=(a, 0, \cdots, 0, a)$ is left invariant. We also recover the correct $S O(9) \stackrel{\langle 16\rangle}{\longrightarrow} S O(7)$ branching rule $16 \rightarrow 8+7+1$ for the spinor field. The linear combinations in (5.8) are thus identified as the three generators of the $S O(7)$ Cartan subalgebra.

After computing the eigenvalues and eigenvectors of $h_{1}, h_{2}$ and $h_{3}$ in the vector irrep, we deduce that the 9 of $S O(9)$ decomposes into $8+1$ under $S O(7)$ as

$$
V_{\mu}=\left(\begin{array}{c}
q_{1}-i q_{8} \\
i q_{1}-q_{8} \\
-q_{4}-i q_{5} \\
-i q_{4}-q_{5} \\
q_{7}+i q_{2} \\
i q_{7}+q_{2} \\
q_{6}-i q_{3} \\
i q_{6}-q_{3} \\
\phi
\end{array}\right) .
$$


Symmetry considerations fix only the relative phases for the spinor components appearing inside this vector. We choose their absolute phases so that the $S O(9)$ hadrons break apart simply into the $S O(7)$ mesons $m^{(i j)}=q^{i^{T}} c q^{j}$ and baryons $b_{i}=\frac{1}{4 !} \epsilon_{i j k l m} q^{j^{T}} \gamma_{\mu} c q^{k} q^{\ell^{T}} \gamma^{\mu} c q^{m}$ :

$$
\begin{aligned}
L & \rightarrow-2 a^{2} \\
M^{(i j)} & \rightarrow 2 i m^{(i j)}+\phi^{i} \phi^{j} \\
N^{i} & \rightarrow-2 a^{2} \phi^{i} \\
R_{i} & \rightarrow 8 a^{2} b_{i} \\
T & \rightarrow 8 a^{2} b_{i} \phi^{i} .
\end{aligned}
$$

Inserting these field decompositions into (5.6), we recover the quantum relation $\operatorname{det} m-$ $b_{i} m^{i j} b_{j}=\Lambda_{7}^{10}$ in Pouliot's model from the first constraint in $W_{N_{f}=5}$ [16], while the second identically vanishes. These results provide strong consistency checks on the $N_{f}=5 S O(9)$ theory.

The ground state structure of the $N_{f} \gtrsim 6 S O(9)$ model can be simply understood in terms of its dual based upon

$$
\widetilde{G}=S U\left(N_{f}-4\right)_{\text {local }} \times\left[S U\left(N_{f}\right) \times U(1)_{Y} \times U(1)_{R}\right]_{\text {global }} .
$$

The magnetic theory has chiral matter content

$$
\begin{aligned}
q_{i}^{\alpha} & \sim\left(\square ; \bar{\square} ; 2, \frac{4}{N_{f}} \frac{N_{f}-5}{N_{f}-4}\right) \\
q^{\prime \alpha} & \sim\left(\square ; 1 ; 0, \frac{N_{f}-5}{N_{f}-4}\right) \\
\bar{q}_{\alpha} & \sim\left(\bar{\square} ; 1 ;-2 N_{f}, \frac{N_{f}-3}{N_{f}-4}\right) \\
s_{\alpha \beta} & \sim\left(\bar{\square} ; 1 ; 0, \frac{2}{N_{f}-4}\right) \\
\ell & \sim\left(1 ; 1 ; 2 N_{f}, 0\right) \\
m^{(i j)} & \sim\left(1 ; \square ;-4,2-\frac{10}{N_{f}}\right) \\
n^{i} & \sim\left(1 ; \square ; 2 N_{f}-2,1-\frac{5}{N_{f}}\right)
\end{aligned}
$$

and tree level superpotential

$$
W_{\text {mag }}=n^{i} q_{i}^{\alpha} \bar{q}_{\alpha}-\ell q^{\prime \alpha} \bar{q}_{\alpha}-m^{(i j)} q_{i}^{\alpha} s_{\alpha \beta} q_{j}^{\beta}+q^{\prime \alpha} s_{\alpha \beta} q^{\prime \beta}-\operatorname{det} s .
$$


Its magnetic beta function

$$
\widetilde{b_{0}}= \begin{cases}4, & N_{f}=6 \\ 2 N_{f}-12, & N_{f} \geq 7\end{cases}
$$

is positive for $6 \leq N_{f}<19$ flavors. The theory then exists at the origin of moduli space in a nonabelian Coulomb phase. All the usual comments regarding anomaly matching, global symmetry agreement and scale simplification hold for this dual like its predecessors. The maps between composite operators in the electric and magnetic theories also follow the familiar pattern. The $R^{[i j k l]}$ and $T^{[i j k l m]}$ hadrons are identified with

$$
\begin{aligned}
& r^{\left[i_{1} \cdots i_{4}\right]}=\epsilon^{i_{1} \cdots i_{N_{f}}} \epsilon_{\alpha_{1} \cdots \alpha_{N_{f}-4}} q_{i_{5}}^{\alpha_{1}} \cdots q_{i_{N_{f}}}^{\alpha_{N_{f}-4}} \\
& t^{\left[i_{1} \cdots i_{5}\right]}=\epsilon^{i_{1} \cdots i_{N_{f}}} \epsilon_{\alpha_{1} \cdots \alpha_{N_{f}-4}} q_{i_{6}}^{\alpha_{1}} \cdots q_{i_{N_{f}}}^{\alpha_{N_{f}-5}} q^{\prime \alpha_{N_{f}-4}},
\end{aligned}
$$

while the electric baryons and exotics

$$
\begin{aligned}
B_{0}^{\left[i_{1} \cdots i_{9}\right]} & =\epsilon^{\mu_{1} \cdots \mu_{9}} V_{\mu_{1}}^{i_{1}} \cdots V_{\mu_{9}}^{i_{9}} \\
B_{1}^{a\left[i_{1} \cdots i_{7}\right]} & =\epsilon^{\mu_{1} \cdots \mu_{9}} V_{\mu_{1}}^{i_{1}} \cdots V_{\mu_{7}}^{i_{7}} W_{\mu_{8} \mu_{9}}^{a} \\
B_{2}^{\left[i_{1} \cdots i_{5}\right]} & =\epsilon^{\mu_{1} \cdots \mu_{9}} V_{\mu_{1}}^{i_{1}} \cdots V_{\mu_{5}}^{i_{5}} W_{\mu_{6} \mu_{7}}^{a} W_{\mu_{8} \mu_{9}}^{a} \\
C_{0}^{\left[i_{1} \cdots i_{8}\right]} & =\epsilon^{\mu_{1} \cdots \mu_{9}} V_{\mu_{1}}^{i_{1}} \cdots V_{\mu_{8}}^{i_{8}} Q^{T} \Gamma_{\mu_{9}} C Q \\
C_{1}^{a\left[i_{1} \cdots i_{6}\right]} & =\epsilon^{\mu_{1} \cdots \mu_{9}} V_{\mu_{1}}^{i_{1}} \cdots V_{\mu_{6}}^{i_{6}} W_{\mu_{7} \mu_{8}}^{a} Q^{T} \Gamma_{\mu_{9}} C Q \\
C_{2}^{\left[i_{1} \cdots i_{4}\right]} & =\epsilon^{\mu_{1} \cdots \mu_{9}} V_{\mu_{1}}^{i_{1}} \cdots V_{\mu_{4}}^{i_{4}} W_{\mu_{5} \mu_{6}}^{a} W_{\mu_{7} \mu_{8}}^{a} Q^{T} \Gamma_{\mu_{9}} C Q
\end{aligned}
$$

are matched onto the magnetic operators

$$
\begin{aligned}
b_{0}^{\left[i_{1} \cdots i_{9}\right]} & =\epsilon^{i_{1} \cdots i_{N_{f}}} \epsilon^{\alpha_{1} \cdots \alpha_{N_{f}-4}}(s q)_{\alpha_{1} i_{10}} \cdots(s q)_{\alpha_{N_{f}-9} i_{N_{f}}}\left(s \widetilde{w}^{a}\right)_{\alpha_{N_{f}-8} \alpha_{N_{f}-7}}\left(s \widetilde{w}^{a}\right)_{\alpha_{N_{f}-6} \alpha_{N_{f}-5}} \bar{q}_{\alpha_{N_{f}-4}} \\
b_{1}^{a\left[i_{1} \cdots i_{7}\right]} & =\epsilon^{i_{1} \cdots i_{N_{f}}} \epsilon^{\alpha_{1} \cdots \alpha_{N_{f}-4}}(s q)_{\alpha_{1} i_{8}} \cdots(s q)_{\alpha_{N_{f}-7} i_{N_{f}}}\left(s \widetilde{w}^{a}\right)_{\alpha_{N_{f}-6} \alpha_{N_{f}-5}} \bar{q}_{\alpha_{N_{f}-4}} \\
b_{2}^{\left[i_{1} \cdots i_{5}\right]} & =\epsilon^{i_{1} \cdots i_{N_{f}}} \epsilon^{\alpha_{1} \cdots \alpha_{N_{f}-4}}(s q)_{\alpha_{1} i_{6}} \cdots(s q)_{\alpha_{N_{f}-5} i_{N_{f}}} \bar{q}_{\alpha_{N_{f}-4}} \\
c_{0}^{\left[i_{1} \cdots i_{8}\right]} & =\epsilon^{i_{1} \cdots i_{N_{f}}} \epsilon^{\alpha_{1} \cdots \alpha_{N_{f}-4}}(s q)_{\alpha_{1} i_{9}} \cdots(s q)_{\alpha_{N_{f}-8} i_{N_{f}}}\left(s \widetilde{w}^{a}\right)_{\alpha_{N_{f}-7} \alpha_{N_{f}-6}}\left(s \widetilde{w}^{a}\right)_{\alpha_{N_{f}-5} \alpha_{N_{f}-4}} \\
c_{1}^{a\left[i_{1} \cdots i_{6}\right]} & =\epsilon^{i_{1} \cdots i_{N_{f}}} \epsilon^{\alpha_{1} \cdots \alpha_{N_{f}-4}}(s q)_{\alpha_{1} i_{7}} \cdots(s q)_{\alpha_{N_{f}-6} i_{N_{f}}}\left(s \widetilde{w}^{a}\right)_{\alpha_{N_{f}-5} \alpha_{N_{f}-4}} \\
c_{2}^{\left[i_{1} \cdots i_{4}\right]} & =\epsilon^{i_{1} \cdots i_{N_{f}}} \epsilon^{\alpha_{1} \cdots \alpha_{N_{f}-4}}(s q)_{\alpha_{1} i_{5}} \cdots(s q)_{\alpha_{N_{f}-4} i_{N_{f}}} .
\end{aligned}
$$

Just as it is possible to recover the confining phase quantum constraint in Pouliot's $S O(7)$ model starting from the $S O(9)$ theory, so can its nonabelian Coulomb phase be reproduced as well. When the spinor vev breaks $S O(9)$ down to $S O(7)$, the $\ell, m^{(i j)}$ and $n^{i}$ 
fields appearing in the first three terms of the magnetic superpotential need to be replaced by their decomposed forms listed in (5.10):

$$
W_{\text {mag }} \rightarrow-2 a^{2} \phi^{i} q_{i}^{\alpha} \bar{q}_{\alpha}+2 a^{2} q^{\prime \alpha} \bar{q}_{\alpha}-\left[2 i m^{(i j)}+\phi^{i} \phi^{j}\right] q_{i}^{\alpha} s_{\alpha \beta} q_{j}^{\beta}+q^{\prime \alpha} s_{\alpha \beta} q^{\prime \beta}-\operatorname{det} s .
$$

Recalling that Pouliot's model contains only spinor matter, we render all the $\phi^{i}$ singlets heavy by introducing additional singlets $\chi_{i}$ and supplementing $W_{\text {mag }}$ with the mass term $W_{\text {tree }}=\mu \phi^{i} \chi_{i}$. The $\chi_{i}$ equation of motion freezes $\phi^{i}$ at zero expectation value. We must

also integrate out $q^{\prime \alpha}$ and $\bar{q}_{\alpha}$ since both acquire mass from the bilinear term in (5.18). The magnetic theory's particle content and superpotential

$$
W_{\mathrm{mag}} \rightarrow-2 i m^{(i j)} q_{i}^{\alpha} s_{\alpha \beta} q_{j}^{\beta}-\operatorname{det} s
$$

thus reduce to those of Pouliot's $\mathrm{SO}(7)$ dual along the spinor flat direction [16].

\section{Conclusion}

The chiral-nonchiral dual pairs which we have examined in this article are related to one another by renormalization group flows along various flat directions. We display the web which connects these four theories to one another as well as to other dual pairs in fig. 1. As can be seen in the figure, Higgsing the electric theory induces mass decoupling in its magnetic counterpart and vice-versa. This basic duality feature was first noted by Seiberg in his dual to supersymmetric QCD [1]. We have repeatedly observed that the complete breaking of the magnetic gauge group and the resulting condensation of magnetic monopoles are correlated with the onset of confinement in the electric theory. This phenomenon is also familiar from SUSY QCD [1]. However, our particular $S O(7), S O(8)$ and $S O(9)$ models possess no analogue of the quantum moduli space that exists in $N_{f}=N_{c}+1$ SUSY QCD. Instead, their nonabelian Coulomb phases give way to confinement characterized by nontrivial quantum relations among colorless moduli. As we have seen, these electric theory quantum constraints can be recovered from semiclassical equations of motion in the magnetic theories. So duality relates complicated nonperturbative phenomena in one description to weakly coupled results in the other.

Several extensions of this work would be interesting to pursue. One could continue to explore further dual pairs which arise along various flat directions of the Pouliot-Strassler $S O(10)$ model. Distler and Karch have recently investigated the long distance emergence 
of accidental global symmetries in duals to $S O(5), S O(3)$ and $S U(3)$ electric theories that appear further down on the web in fig. 1 [12]. Studies similar to theirs and ours could be undertaken for other examples of chiral-nonchiral dual pairs. But clearly, it would be preferable to generalize all these results and find new duals which reduce to those displayed in the figure as special cases. Obvious starting points to consider include $S O(9)$ and $S O(10)$ with two spinors and $N_{f}$ vector flavors as well as $S O(11)$ with one spinor and various numbers of vectors [22]. As we noted in our opening remarks, there unfortunately exists no systematic field theory method for deducing magnetic counterparts to these larger electric theories. But they must at least reduce to those which we have analyzed in this paper along certain flat directions. It seems likely that such bigger dual pairs also extend the patterns and trends which we repeatedly encountered in this work. So we hope our findings will provide useful clues in future duality searches.

\section{Acknowledgments}

It is a pleasure to thank Howard Georgi, Matt Strassler and especially Per Kraus for many helpful discussions. 


\section{References}

[1] N. Seiberg, Nucl. Phys. B435 (1995) 129.

[2] K. Intriligator and P. Pouliot, Phys. Lett. B353 (1995) 471.

[3] K. Intriligator and N. Seiberg, Nucl. Phys. B444 (1995) 125.

[4] D. Kutasov, Phys. Lett. B351 (1995) 230.

[5] D. Kutasov and A. Schwimmer, Phys. Lett. B354 (1995) 315.

[6] K. Intriligator, Nucl. Phys. B448 (1995) 187.

[7] R.G. Leigh and M.J. Strassler, Nucl. Phys. B447 (1995) 95.

[8] K. Intriligator, R.G. Leigh and M.J. Strassler, Nucl. Phys. B456 (1995) 567.

[9] D. Kutasov, A. Schwimmer and N. Seiberg, Nucl. Phys. B459 (1996) 455.

[10] J. Brodie, Nucl. Phys. B478 (1996) 123.

[11] P. Ramond, hep-th 9608077, unpublished.

[12] J. Distler and A. Karch, hep-th 9611088, unpublished.

[13] C. Csàki, M. Schmaltz, W. Skiba and J. Terning, hep-th 9701191, unpublished.

[14] M. Bershadsky, A. Johansen, T. Pantev, V. Sadov and C. Vafa, hep-th 9612052, unpublished.

[15] C. Vafa and B. Zweibach, hep-th 9701015, unpublished.

[16] P. Pouliot, Phys. Lett. B359 (1995) 108.

[17] P. Pouliot and M. Strassler, Phys. Lett. B370 (1996) 76.

[18] P. Pouliot and M. Strassler, Phys. Lett. B375 (1996) 175.

[19] T. Kawano, Prog. Theor. Phys. 95 (1996) 963.

[20] M.A. Shifman and A.I. Vainshtein, Nucl. Phys. B359 (1991) 571.

[21] A.G. Elashvili, Funk. Anal. Pril. 6 (1972) 51.

[22] P. Cho, hep-th 9701020, unpublished. 


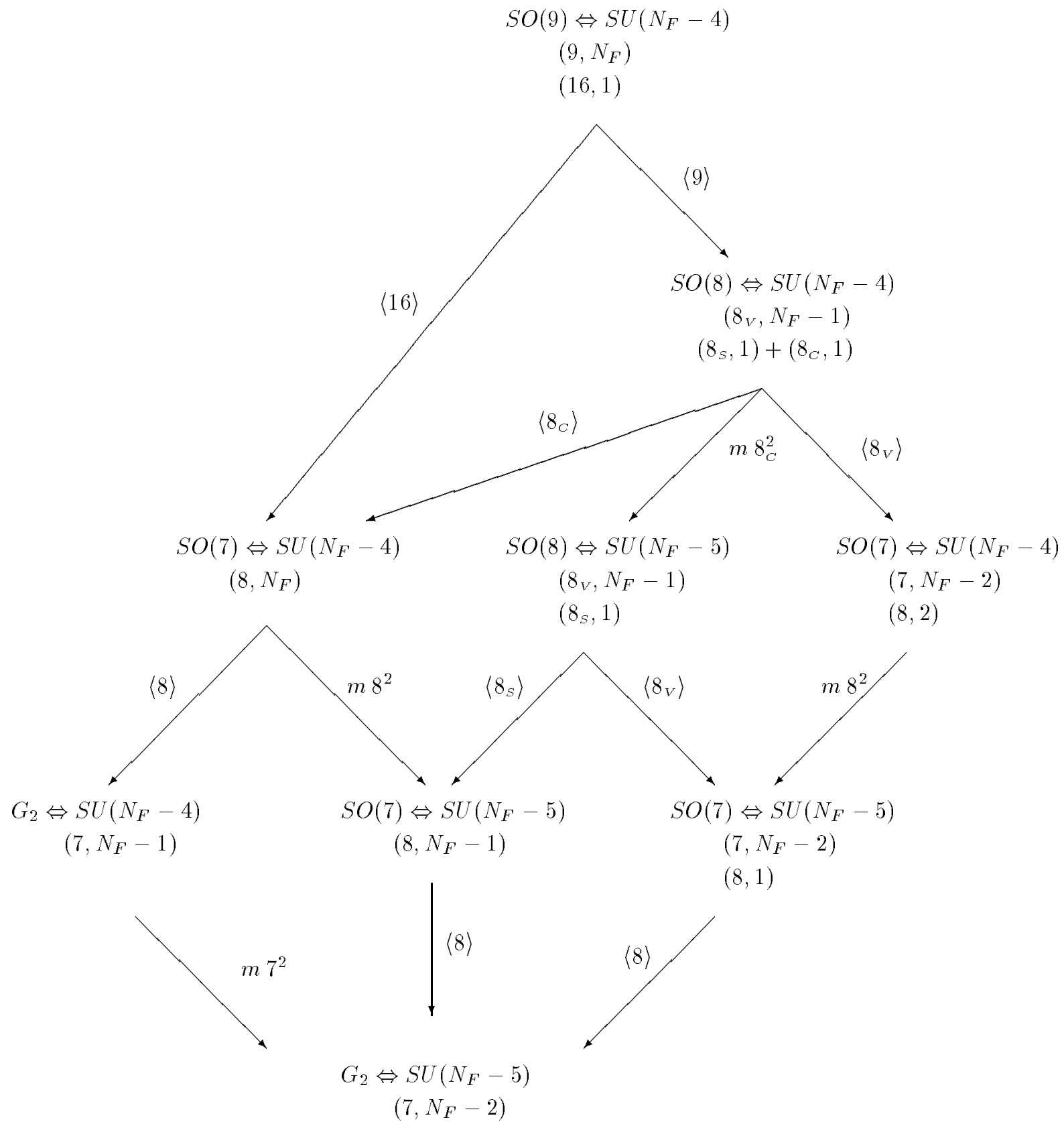

Fig 1. Schematic diagram which illustrates renormalization group flows among various chiral-nonchiral dual pairs. Dual electric and magnetic gauge groups are indicated by short broad double arrows. Superfield matter contents in each electric theory are also displayed. Flows along electric (magnetic) flat directions are depicted by long thin arrows with vev (mass term) annotations. 